\title{
Bases para el abordaje multidisciplinario de la seudo paresia y seudo parálisis de hombro por patología del manguito rotador
}

\author{
Basis for the multidisciplinary approach to shoulder pseudoparesis \\ and pseudoparalysis due to rotator cuff pathology
}

\author{
Santos-Zaldívar KP,* López-Muñoz R, * Aguiñaga-Malanco SE, ${ }^{\ddagger}$ Méndez-Domínguez N \\ Universidad Marista de Mérida. Mérida, Yucatán, México.
}

RESUMEN. Introducción: El hombro doloroso es una entidad que genera gran parte de las consultas de ortopedia a nivel mundial, si bien puede afectar en cualquier etapa de la vida, su prevalencia es mayor conforme aumenta la edad. La afección del manguito rotador suele ser la causa más relevante de dolor y disfunción. Dos consecuencias comunes de la afección del manguito rotador son la seudo paresia y la seudo parálisis y aun cuando comparten una etiología y sintomatología semejantes, su diferenciación clínica es fundamental para su manejo óptimo y resolución oportuna. Objetivo: Revisar las bases teóricas y clínicas para la diferenciación y manejo de la seudo paresia y seudo parálisis por patología del manguito rotador. Material y métodos: Revisión descriptiva de los conceptos de seudo paresia y seudo parálisis desde la perspectiva ortopédica, de fisioterapia y rehabilitación. Resultados: Una vez que se comprenden las diferencias en los mecanismos de lesión del manguito rotador y el modo en el que se traducen en la semiología del hombro doloroso, es posible integrar un diagnóstico diferencial entre seudo paresia y seudo parálisis. El diagnóstico debe integrar las evaluaciones y ejercicios específicos para que permitan identificar ambas condiciones $\mathrm{y}$, una vez que se han distinguido e identificado, permitirá orientar el abordaje terapéutico de manera óptima. Conclusión: La diferenciación entre seudo parálisis y seudo paresia permite comprender los mecanismos de lesión y, finalmente, pro-
ABSTRACT. Introduction: The painful shoulder is an entity that generates much of the orthopedic consultations worldwide, although it can affect at any stage of life, its prevalence is higher as age increases. Rotator cuff condition is often the most relevant cause of pain and dysfunction. Two common consequences of rotator cuff condition are pseudoparesis and pseudoparalysis, and even though they share similar etiology and symptomatology, their clinical differentiation is critical for optimal management and timely resolution. Objective: To review the theoretical and clinical bases for the differentiation and management of pseudoparesis and pseudoparalysis due to rotator cuff pathology. Material and methods: Descriptive review of the concepts of pseudoparesis and pseudoparalysis from the orthopedic, physiotherapy and rehabilitation perspective. Results: Once the differences in rotator cuff injury mechanisms and how they translate into painful shoulder semiology are understood, it is possible to integrate a differential diagnosis between pseudoparesia and pseudoparalysis. Diagnosis should integrate specific evaluations and exercises to identify both conditions and, once distinguished and identified, allow optimal guidance of therapeutic approach. Conclusion: The differentiation between pseudoparalysis and pseudoparesis allows us to understand the mechanisms of injury and, finally, to

\section{Nivel de evidencia: $\mathrm{V}$}

* Escuela de Medicina.

₹ Escuela de Fisioterapia y Rehabilitación.

Dirección para correspondencia:

Nina Méndez-Domínguez

Escuela de Medicina. Campus de Ciencias de la Salud. Universidad Marista de Mérida.

Periférico Norte Tablaje Catastral 13941, Carretera Mérida-Progreso, CP 97300, Mérida, Yucatán, México.

E-mail: ninuxka@hotmail.com

Citar como: Santos-Zaldívar KP, López-Muñoz R, Aguiñaga-Malanco SE, Méndez-Domínguez N. Bases para el abordaje multidisciplinario de la seudo paresia y seudo parálisis de hombro por patología del manguito rotador. Acta Ortop Mex. 2020; 34(4): 242-248. https://dx.doi.org/10.35366/97559 
porcionar el tratamiento óptimo a los pacientes con hombro doloroso por patología del manguito rotador.

Palabras clave: Lesión, manguito rotador, seudo paresia, diagnóstico diferencial, hombro.

\section{Introducción}

El hombro doloroso es una de las afecciones musculoesqueléticas más frecuentes, con una prevalencia estimada entre 6.7 y $66.7 \%$ de la población en general, lo que significa uno de los principales motivos de consulta. Del 40 al 51\% de las personas presentarán hombro doloroso en algún momento de su vida; por lo cual, después de los 65 años su prevalencia es de $25 \% .^{1,2,3,4}$

El hombro doloroso es un evento multifactorial cuyas causas van desde la patología del manguito de los rotadores hasta el atrapamiento de nervios periféricos. ${ }^{1}$ Sin embargo, se ha descrito que los trastornos del manguito rotador son la causa más común de patología en hombro, lo que reresenta casi 50\% de las lesiones mayores que ocurren en él.,

Por su parte, la ruptura del manguito rotador es causa importante de dolor y disfunción en el hombro. ${ }^{7,8,9,10}$ La prevalencia de los desgarros masivos del manguito rotador varía en la literatura entre 20 y 40\%; no obstante, conforman aproximadamente $80 \%$ de todos los casos de recurrencia en el desgarro del maguito rotador. ${ }^{11,12,13}$

La ruptura masiva del manguito rotador se encuentra asociada con la presentación de dos condiciones clínicas que son la seudo paresia y la seudo parálisis del deltoides. El término «paresia» hace referencia a la presencia de debilidad con algún grado de movimiento, mientras la parálisis se puede definir como la pérdida completa del movimiento. ${ }^{14}$

El presente artículo tiene el objetivo de revisitar las bases teóricas y clínicas para la diferenciación y manejo de la seudo paresia y seudo parálisis por patología del manguito rotador.

\section{Material y métodos}

Revisión descriptiva de los conceptos de seudo paresia y seudo parálisis desde la perspectiva ortopédica, de fisioterapia y rehabilitación. Con la finalidad de esquematizar los mecanismos de lesión, se ofrece al lector las representaciones anatómicas y fisiopatológicas de las entidades aquí descritas. Para orientar el diagnóstico diferencial se integran las evaluaciones y pruebas de orientación diagnóstica con sus correspondientes descripciones.

\section{Resultados}

Es relevante ubicarse en la anatomía del hombro para el entendimiento de la relación entre la ruptura masiva e irreparable del manguito de los rotadores y la afectación de la funcionalidad del hombro para establecer el vínculo con la provide the optimal treatment for patients with painful shoulder due to rotator cuff pathology.

Keywords: Injury, rotator cuff, pseudoparesia, differential diagnosis, shoulder.

presentación de seudo paresia y seudo parálisis. ${ }^{15,16,17}$ Está constituida por varias articulaciones: esternoclavicular, acromioclavicular, glenohumeral y escapulotorácica. ${ }^{18}$ En conjunto, estas articulaciones trabajan a un ritmo sincronizado para permitir el rango de movimiento del hombro. ${ }^{19}$

La articulación escapulohumeral es la más importante. Es una articulación esférica o enartrosis. La superficie articular glenoidea es más gruesa en la periferia, formando el rodete glenoideo, tiene el mayor grado de libertad de movimiento, pero se consigue a expensas de la estabilidad.

Los músculos escapulohumerales son los llamados músculos intrínsecos del hombro. Son un grupo de seis músculos que convergen desde la escápula hasta el húmero y rodean la articulación escapulohumeral: deltoides, redondo mayor, supraespinoso, infraespinoso, redondo menor y subescapular. Estos músculos son relativamente cortos y actúan sobre la articulación del hombro. El deltoides es un potente y grueso músculo que cubre el hombro y forma su contorno redondeado. Tiene forma de letra griega delta invertida. Se divide en dos porciones unipenniformes (anterior o clavicular y posterior o espinal) y una multipenniforme (medial o acromial), que pueden actuar separadamente o como un todo. El redondo mayor es un músculo grueso redondeado que discurre lateralmente al tercio inferolateral de la escápula. ${ }^{20}$ Los otros cuatro músculos forman en conjunto el manguito de los rotadores, son el grupo más profundo de la articulación del hombro, formando una unidad funcional única que involucra la cabeza humeral, colaborando con la estabilidad de la articulación glenohumeral y en los movimientos realizados por el miembro superior. ${ }^{17,21}$ La contracción tónica de estos músculos sujeta la cabeza del húmero en la pequeña y poco profunda cavidad glenoidea de la escápula durante los movimientos del brazo. ${ }^{17}$

Otra relación anatómica importante del hombro es el plexo braquial, el cual pasa por debajo de la clavícula; al ingresar en la axila las divisiones se reagrupan alrededor de la arteria axilar, lo que origina las tres cuerdas del plexo: lateral, posterior y medial. En su trayecto hacia la axila, las cuerdas atraviesan el borde lateral del músculo pectoral menor y se dividen en los nervios terminales que inervan toda la extremidad superior: nervio mediano, nervio cubital, nervio musculocutáneo, nervio braquial cutáneo interno y su accesorio. ${ }^{22}$

La capacidad del hombro para múltiples grados de movimiento se encuentra basada en la interacción de diversas estructuras que reaccionan a los estímulos mecánicos, teniendo un adecuado ajuste. Las estructuras óseas, el labrum fibrocartilaginoso, la cápsula articular y los ligamentos glenohumerales incrementan la estabilidad del hombro. No obstante, 
dichas estructuras estabilizadoras estáticas se encuentran respaldadas por el conjunto de músculos que rodean la cintura escapular, esto brinda una estabilidad dinámica articular. Los músculos del manguito rotador (MMR) no solamente actúan como estabilizadores activos, sino que también refuerzan la estabilidad pasiva debido a su ubicación y orientación alrededor de la articulación glenohumeral. ${ }^{23,24}$

Los estabilizadores estáticos y dinámicos reaccionan a las fuerzas externas aplicadas a la articulación glenohumeral para proporcionar estabilidad en diferentes posiciones durante el arco de movimiento (ROM). La articulación escapulotorácica proporciona al hombro grados de movilidad adicionales y contribuye a la estabilidad articular. La combinación de estos factores da lugar a un sistema biomecánico complejo con la capacidad de adaptarse y responder a las necesidades de la extremidad superior; ${ }^{23,24}$ sin embargo, cuando existe anomalía alguna en los estabilizadores estáticos o dinámicos de la articulación glenohumeral se afecta la función del hombro, lo que puede desencadenar trastornos importantes. ${ }^{25}$ Los músculos del manguito rotador se encuentran en posiciones específicas y concretas para resistir el estrés continuo de la articulación glenohumeral en conjunto con los ligamentos capsulares. Cada músculo, individualmente, tiene acciones (Tabla 1) y funciones independientes que, al combinarse, contribuyen a la estabilización global de la articulación durante los rangos intermedios y finales del movimiento. ${ }^{26}$

El manguito rotador se puede considerar como un sistema muscular de control fino que se ajusta mediante la retroalimentación neuromuscular de las fuerzas generadas durante el ROM y por la respuesta de los ligamentos glenohumerales. Así mismo, los MMR ejercen una fuerza de compresión a lo largo de la articulación glenohumeral,

Tabla 1: Acción de los músculos del manguito rotador.

\begin{tabular}{|c|c|c|}
\hline Músculo & Acción & $\begin{array}{c}\text { Grados de } \\
\text { movilidad } \\
\text { articular }\end{array}$ \\
\hline Infraespinoso & Rotación externa del hombro & $90^{\circ}$ \\
\hline Supraespinoso & Abducción del hombro & $180^{\circ}$ \\
\hline Redondo menor & Rotación externa del hombro & $90^{\circ}$ \\
\hline Subescapular & $\begin{array}{l}\text { Rotación externa del hombro } \\
\text { (completa extensión) }\end{array}$ & $\begin{array}{c}\text { Rotación } \\
\text { externa: } 90^{\circ}\end{array}$ \\
\hline
\end{tabular}

manteniendo la cabeza humeral en lo más profundo de la concavidad de la glenoides, disminuyendo las fuerzas y centralizando la cabeza humeral en la glenoides. . $^{25,27,28}$

La patología del manguito rotador puede deberse al pinzamiento, la ruptura parcial, completa, masiva y la artropatía por lesión masiva del manguito rotador. La etiología de la ruptura del manguito rotador se relaciona con factores extrínsecos (originados en estructuras que rodean al tendón) e intrínsecos (originados dentro del tendón). ${ }^{25}$

Entre los factores extrínsecos que han sido relacionados están las variaciones del arco coracoacromial y la forma del acromion. En la actualidad los factores intrínsecos son los más importantes en relación con la etiología, entre ellos la degeneración por envejecimiento parece ser el más relevante, debido al descenso en el flujo sanguíneo que sufre el tendón del músculo supraespinoso con respecto a la edad. ${ }^{29}$

Existen varios mecanismos que producen la ruptura o lesión del manguito rotador como el microtrauma repetitivo o el uso excesivo en la realización de movimientos repetitivos por arriba de la cabeza, ya sea por actividades laborales o deportivas. . $31,32^{3}$ Otro mecanismo de lesión es el traumático, producido por caídas en donde se empleó el brazo como amortiguador (Tabla 2). ${ }^{4}$

Las rupturas del manguito de los rotadores se pueden dividir según su espesor en parcial, total y masivas. Las parciales afectan parte del espesor del tendón, se clasifican según la localización anatómica y la profundidad de la lesión, ya sea en milímetros o en porcentaje. ${ }^{29}$ Las rupturas totales son las que afectan el espesor total del tendón, pueden clasificarse según su morfología en «U», «L», «L invertida» o en semiluna. El reconocimiento del patrón de ruptura es importante para la planificación quirúrgica. ${ }^{12,27,30,33,34,35}$

Los desgarros masivos no son sinónimo de rupturas irreparables. Los signos de que el desgarre es irreparable incluyen una migración superior estática de la cabeza del húmero, un intervalo acromiohumeral estrecho o ausente e infiltración grasa que afecta más o menos de $50 \%$ de la musculatura del manguito de los rotadores. ${ }^{12,32}$

Los desgarros crónicos masivos del manguito de los rotadores pueden asociarse con una discapacidad dolorosa del hombro con o sin pérdida del rango de movimiento activo y función éste. Sin embargo, la pérdida del rango de movimiento activo que se presente en algunos pacientes puede restringir considerablemente su actividad. ${ }^{12,36}$ Cuando existe
Compresión primaria

Compresión secundaria Tensión

Traumatismos agudos
Efecto producido por la compresión extrínseca del arco coracoacromial y la degeneración tendinosa por envejecimiento. Se presenta con mayor frecuencia en pacientes mayores de 40 años Ocasionada secundaria a inestabilidad glenohumeral anterior. Se presenta en pacientes jóvenes Producida por microtraumatismos repetidos durante la fase de desaceleración, que ocasiona una sobrecarga excéntrica en el manguito. Se visualizan desgarros en la cara inferior del manguito y lesiones del complejo rodete-inserción del bíceps. Se puede presentar en deportistas o en personas que realizan trabajos con movimiento del brazo por encima de la cabeza

Debido a traumatismos de alta energía o en deportes de contacto. El mecanismo de aducción forzada y abducción activa contra resistencia es el más frecuente 

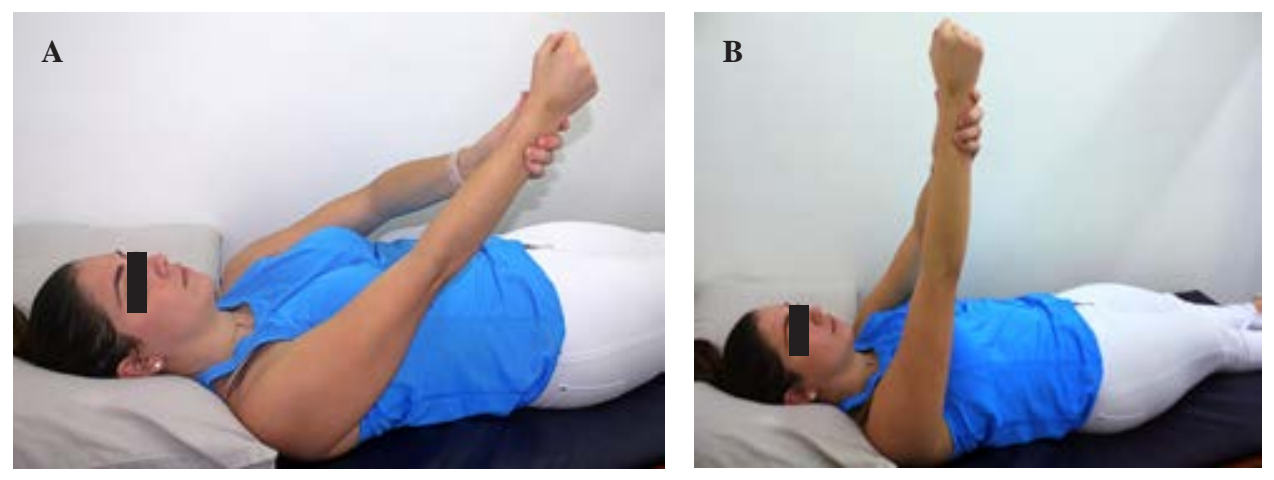

Figura 1: Programa de ejercicios para fortalecer el deltoides. A) Paciente en decúbito supino, con una almohada colocada debajo de su cabeza. Levantando el brazo lesionado de forma vertical a $90^{\circ}$ con la ayuda de su otro brazo si fuera necesario. Con el codo en extensión y en alineación con la oreja del paciente. B) Paciente manteniendo la posición vertical de su brazo lesionado con su propia fuerza.



Figura 2: Programa de ejercicios para fortalecer el deltoides. Ejercicio 3. El paciente realizará el ejercicio anterior desde el primer paso (ejercicio 2) añadiendo un ligero peso (aproximadamente $250 \mathrm{~g}$ ). Se debe realizar cuando el paciente ya tenga confianza de la movilidad de su hombro.

pérdida del movimiento activo se puede deber a dos condiciones denominadas seudo parálisis y seudo paresia, por lo cual es importante definir ambos conceptos, ya que el tratamiento y los pronósticos son diferentes. ${ }^{16}$

La seudo paresia es la condición en donde los pacientes alcanzan una elevación activa de menos de $90^{\circ}$ con una elevación pasiva libre. ${ }^{37}$ La seudo parálisis hace referencia a la condición de los pacientes que tienen $0^{\circ}$ de elevación activa, usualmente asociada con un escape anterosuperior de la cabeza del húmero, de forma estática o dinámica. ${ }^{16,38}$

Las pruebas clínicas nos permiten diferenciar las dos condiciones, estas pruebas consisten en solicitar al paciente que realice una elevación activa del hombro y, posteriormente, evaluar su movilidad pasiva. Es necesario considerar la aplicación de una inyección de lidocaína al momento de realizar las pruebas, ya que el dolor puede ser un impedimento para la realización correcta de las pruebas, afectando la sensibilidad y especificidad de éstas. ${ }^{9,39}$

El tratamiento inicial consiste en reposo y en la modificación de la actividad física para evitar flexiones del hombro superiores a los 90 grados. Lo anterior asociado a un trata- miento analgésico y antiinflamatorio, con el fin de disminuir el dolor e iniciar un programa de rehabilitación precoz para recuperación del movimiento. ${ }^{4,29}$

El dolor se trata según la escalera analgésica de la Organización Mundial de la Salud (OMS), para así determinar los fármacos más adecuados para su episodio de dolor. ${ }^{40,41}$

Se ha propuesto a los antiinflamatorios no esteroideos (AINE) como la base para el control del dolor junto con un fármaco coadyuvante al tratamiento..$^{42,43,44}$ Se deben conocer las peculiaridades fisiológicas del paciente para el correcto manejo y control analgésico del dolor con los mínimos riesgos. ${ }^{45}$

Además del tratamiento farmacológico, se debe recomendar al paciente evitar movimiento, posturas y situaciones que propicien la aparición del dolor. ${ }^{46}$

Se considera pertinente recomendar al paciente que evite movimientos repetitivos del hombro doloroso o movimientos prolongados del miembro superior por encima de la línea horizontal y movimientos bruscos. En cuanto a las actividades diarias, se debe recomendar al paciente ponerse la ropa comenzando por el hombro doloroso y evitar extender el miembro superior hacia atrás y al centro de la espalda (rotación medial forzada). ${ }^{46,47}$

\section{Tratamiento de la seudo paresia}

Se ha establecido a la fisioterapia como manejo de elección en el tratamiento de la seudo paresia. ${ }^{16}$ El objetivo es el fortalecimiento de la musculatura deltoidea y pericapsular; ${ }^{34,48}$ sin embargo, el grado de recuperación funcional del miembro superior en general, y del hombro en particular, dependerá de las expectativas, actividades básicas e instrumentales de la vida diaria del paciente. ${ }^{46}$

Levy describe el sistema de rehabilitación y reeducación del músculo deltoides anterior para compensar la deficiencia del manguito rotador. Este enfoque se basa en estudios biomecánicos que demuestran el papel importante que tiene el deltoides anterior para la prevención de la migración superior de la cabeza del húmero y la compresión de la articulación glenohumeral en presencia de un desgarro importante del manguito rotador. ${ }^{12,49}$ Los ejercicios iniciales 
para la rehabilitación del deltoides anterior se realizan con el paciente en decúbito supino y con la cabeza apoyada en una almohada (Figura 1). El paciente es instruido para que lleve el brazo a la posición vertical e intente mantenerlo con la fuerza de contracción del deltoides, eliminando la gravedad dentro de un arco cómodo, el cual irá aumentando en la medida en que el paciente gane confianza. Los ejercicios de esta primera etapa se realizarán de tres a cinco veces al día durante las primeras seis semanas.

La siguiente fase consisten en que el paciente repita el arco gradualmente con un pequeño peso (Figuras 2 y 3). ${ }^{49}$ La duración total de este programa debe ser de mínimo 12 semanas. Los ejercicios deben suspenderse si el dolor aumenta y es necesario reevaluar al paciente a las seis y 12 semanas (Figura 4). ${ }^{50}$

Gutiérrez-Espinoza integra como parte del tratamiento para la ruptura masiva del manguito rotador a la terapia manual y ejercicios específicos de control muscular del hombro y escápula durante 12 semanas, con dos sesiones a la semana. La terapia manual consiste en dos técnicas: movilización glenohumeral posterior y movilización escapular (Tabla 3). ${ }^{51}$

Al culminar la fase de terapia manual se debe proseguir con los ejercicios, considerando los siguientes principios: los ejercicios no deben de producir dolor ( $<4 / 10$ en EVA), comenzar con autocargas o cargas bajas no mayores a $90^{\circ}$, no realizar más de cuatro ejercicios por sesión y es necesario hacer énfasis en la calidad del ejercicio (control motor), el cual debe realizarse de forma lenta, consciente y progresiva. ${ }^{52}$

Otro elemento para considerar en el abordaje del hombro es la activación de los estabilizadores escapulares (trapecio superior, medio e inferior, romboides, elevador de la escápula, serrato anterior y pectoral mayor), debido a que los individuos con hombro doloroso presentan anomalías a nivel de la cinemática escapular. Esta anormalidad puede estar relacionada con la debilidad de la musculatura periescapular, específicamente con la activación excesiva del trapecio superior y la inhibición en la activación del trapecio inferior y serrato anterior. ${ }^{52}$

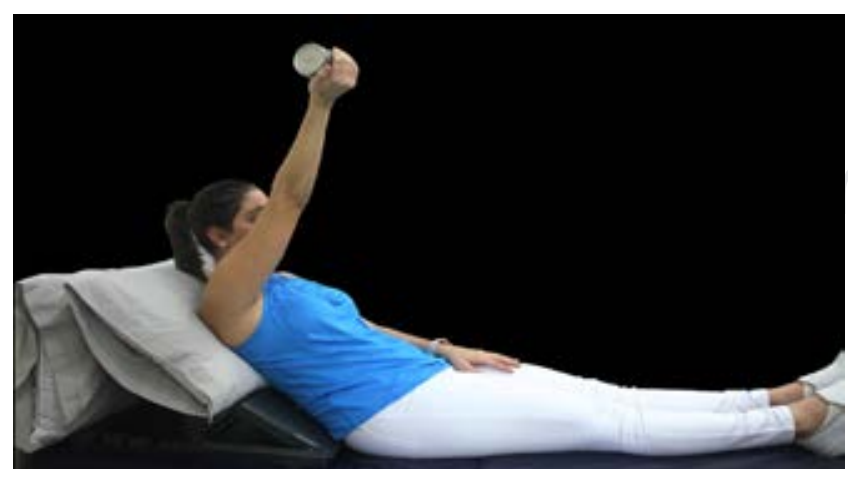

Figura 3: Se realiza en posición Fowler. El paciente debe realizar el ejercicio 2 contra gravedad. Primero sin ningún peso y luego utilizando el mismo peso que se empleó en la posición decúbito supino.
En resumen, el tratamiento deberá incluir manejo del dolor mediante el uso de medios físicos, así como reestablecimiento del control neuromuscular, fuerza muscular, movilidad y estabilidad ortostática y del equilibrio. ${ }^{53,54}$

\section{Manejo de la seudo parálisis}

El abordaje quirúrgico es el tratamiento de elección para revertir la seudo parálisis, tanto en pacientes mayores como en menores de 65 años, por lo que se han propuesto diferentes procedimientos quirúrgicos, entre los cuales destacan la artroplastía total de hombro de anatomía reversa, la cual tiene como principio biomecánico la mundialización y estabilización del centro de rotación de la articulación del hombro, con lo cual reestablece su rango de movimiento y funcionalidad. ${ }^{5}$

Otra opción terapéutica es la transferencia muscular, la cual es efectiva en los adultos mayores si se combina con la artroplastía total de hombro de anatomía reversa; pero no se recomienda como alternativa de tratamiento en la población joven, debido a que requiere una mayor demanda funcional; por lo cual, el objetivo del tratamiento en este caso es la colocación anatómica del manguito rotador en la tuberosidad mayor. ${ }^{16,56}$

En pacientes con ausencia de enfermedad degenerativa de la articulación, es necesario evaluar condiciones como la edad y el grado de lesión del manguito de los rotadores, ya que en pacientes jóvenes en los que el tejido restante es de buena calidad sin cambios de Goutallier, es posible que los procedimientos de tejidos blandos como la reparación del manguito, los parches de aumento o la RCS (reconstrucción capsular superior) sean útiles para la restauración del movimiento. ${ }^{9}$ Esta última opción puede preservar la articulación glenohumeral en pacientes con desgarros irreparables del manguito rotador. La RCS agrega una resistencia biológica, pasiva y superior a la articulación glenohumeral, con lo cual optimiza las parejas de fuerza del manguito rotador y mejora la cinética de la articulación.

Finalmente, la artroplastía reversa de hombro ha mostrado mejores resultados, con un rango mayor de movimiento posterior a su implantación. Además, en pacientes con en-

Tabla 3: Movilizaciones empleadas

en la fisioterapia del hombro doloroso.

Movilización glenohumeral posterior Movilización escapular

Posicionar al paciente en decúbito supino con abducción de 30-40 y ligera rotación externa Realizar una tracción axial inferior y posteriormente un deslizamiento posterior mantenido durante 1 minuto

Dosificación para ambas técnicas: - De 8 a 10 repeticiones por técnica con 30 segundos de descanso entre cada una 



Figura 4: Fortalecimiento del deltoides. Paciente en bipedestación, con la mano en puño del lado afectado y con la palma de la mano del lado opuesto ejerciendo resistencia. El paciente deberá ir elevando el brazo afectado manteniendo la resistencia contralateral, con el fin de que pueda elevar el brazo afectado por arriba de su cabeza. Se deben repetir los ejercicios con el objetivo de que el músculo deltoides realice contracción concéntrica, incluso sin la oposición del brazo no lesionado.

fermedad degenerativa articular importante podría ser el tratamiento más confiable para devolver la funcionalidad del hombro sin presencia de dolor. ${ }^{16}$

\section{Discusión}

En la actualidad no existe una correcta diferenciación de las condiciones de seudo parálisis y seudo paresia en la práctica clínica médica y fisioterapéutica, ya que ambos términos apenas fueron definidos por Tokish en el 2017. Esto representa un reto, puesto que es necesario difundir ampliamente ambos conceptos en las poblaciones de médicos ortopedistas y fisioterapeutas, debido a que el correcto empleo de ambos términos en la práctica médica permitirá un adecuado diagnóstico y tratamiento para cada condición en específico, con lo cual se favorece el pronóstico del paciente, puesto que será tratado en función a su padecimiento, lo cual es relevante por ser una patología, correctamente diagnosticada y tratada tendrá un pronóstico mejor y, a su vez, se traducirá en una mejor calidad de vida para los pacientes.

\section{Conclusión}

La seudo paresia y seudo parálisis al diferenciarse únicamente por el grado de movilidad activa, pueden ser términos confusos, pero los tratamientos son diferentes, porque, en el caso de la seudo paresia, se establece a la fisioterapia como el tratamiento de elección. A diferencia de la seudo parálisis, en donde se propone a la artroplastía inversa de hombro. En lo anterior radica la importancia de diferenciar ambas condiciones, difundirlas y que sean aplicadas en la práctica clínica, ya que el correcto diagnóstico y tratamiento impactarán en el rango de movilidad que pueda tener el paciente posterior a su tratamiento, lo cual es de suma relevancia, ya que tiene un impacto en su funcionalidad y en la realización de sus actividades diarias.

Bibliografía

1. Chang KV, Mezian K, Naňka O, Wu WT, Lin CP, Özçakar L. Ultrasound-guided interventions for painful shoulder: from anatomy to evidence. J Pain Res. 2018; 11: 2311-22. doi: 10.2147/JPR. S169434.

2. Pogorzelski J, Horan MP, Hussain ZB, Vap A, Fritz EM, Millett PJ. Subpectoral biceps tenodesis for treatment of isolated type II SLAP lesions in a young and active population. Arthroscopy. 2018; 34(2): 371-6.

3. Mas G, Barraquer ME. Hombro doloroso. JANO [Internet]. 2006; 615(1). Disponible en: http://www.jano.es/ficheros/sumarios/1/0/161 5/46/1v0n1615a13090785pdf001.pdf

4. Valero Flores N, Navarro García N, Ruiz Caballero JA, Jiménez Díaz JF, Brito Ojeda ME. Lesión del manguito de los rotadores. Canarias Médica y Quirúrgica. 2007; 5(13): 55-65. Disponible en: https:// accedacris.ulpgc.es/bitstream/10553/5992/1/0514198_00013_0009.pdf

5. Tashjian RZ, Southam JD, Clevenger T, Bachus KN. Biomechanical evaluation of graft fixation techniques for acromioclavicular joint reconstructions using coracoclavicular tendon grafts. J Shoulder Elbow Surg. 2012; 21(11): 1573-9. doi: 10.1016/j.jse.2011.10.029.

6. Murrell G, Bhargav D. Shoulder stiffness: diagnosis. Aust Fam Physician. 2004; 33(3): 143-7.

7. Yamamoto A, Takagishi K, Osawa T, Yanagawa T, Nakajima D, Shitara $\mathrm{H}$, et al. Prevalence and risk factors of a rotator cuff tear in the general population. J Shoulder Elbow Surg. 2010; 19(1): 116-20.

8. Mihata T, McGarry MH, Tibone JE, Fitzpatrick MJ, Kinoshita M, Lee TQ. Biomechanical assessment of Type II superior labral anteriorposterior (SLAP) lesions associated with anterior shoulder capsular laxity as seen in throwers: a cadaveric study. Am J Sports Med. 2008; 36(8): 1604-10. doi: 10.1177/0363546508315198.

9. Sellers TR, Abdelfattah A, Frankle MA. Massive rotator cuff tear: when to consider reverse shoulder arthroplasty. Curr Rev Musculoskelet Med. 2018; 11(1): 131-40. doi: 10.1007/s12178-0189467-2.

10. Tempelhof S, Rupp S, Seil R. Age-related prevalence of rotator cuff tears in asymptomatic shoulders. J Shoulder Elbow Surg. 1999; 8(4): 296-9.

11. Denard PJ, Lädermann A, Jiwani AZ, Burkhart SS. Functional outcome after arthroscopic repair of massive rotator cuff tears in individuals with pseudoparalysis. Arthroscopy. 2012; 28(9): 1214-9. doi: 10.1016/j.arthro.2012.02.026.

12. Bedi A, Kovacevic D, Hettrich C, Gulotta LV, Ehteshami JR, Warren $\mathrm{RF}$, et al. The effect of matrix metalloproteinase inhibition on tendonto-bone healing in a rotator cuff repair model. J Shoulder Elbow Surg. 2010; 19(3): 384-91. doi: 10.1016/j.jse.2009.07.010.

13. Lädermann A, Denard PJ, Boileau P, Farron A, Deransart P, Terrier A, et al. Effect of humeral stem design on humeral position and range of motion in reverse shoulder arthroplasty. Int Orthop. 2015; 39(11): 2205-13. doi: 10.1007/s00264-015-2984-3.

14. Thigpen CA, Shanley E, Momaya AM, Kissenberth MJ, Tolan SJ, Tokish JM, et al. Validity and responsiveness of the single alphanumeric evaluation for shoulder patients. Am J Sports Med. 2018; 46(14): 3480-5. doi: 10.1177/0363546518807924. 
15. Donnelly TD, Ashwin S, MacFarlane RJ, Waseem M. Suppl 3: Clinical assessment of the shoulder. Open Orthop J. 2013; 7: 310-5. doi: 10.2174/1874325001307010310.

16. Tokish JM, Alexander TC, Kissenberth MJ, Hawkins RJ. Pseudoparalysis: a systematic review of term definitions, treatment approaches, and outcomes of management techniques. J Shoulder Elbow Surg. 2017; 26(6): e177-87. doi: 10.1016/j.jse.2017.02.024.

17. Di Giacomo G, Pouliart N, Costantini A, De Vita A. Atlas of functional shoulder anatomy. Italia: Springer Science \& Business Media; 2008.

18. Culham E, Peat M. Spinal and shoulder complex posture. I: measurement using the 3Space Isotrak. Clin Rehabil. 1993; 7(4): 309-18.

19. Zorzetto AA, Urban LABD, Liu CB, Prevedello L, Zapparoli M, Vitola MLA, et al. A ecografia no diagnóstico das lesões músculotendinosas do ombro. Radiol Bras. 2003; 36(4): 237-42.

20. Pearl ML. Proximal humeral anatomy in shoulder arthroplasty: Implications for prosthetic design and surgical technique. J Shoulder Elbow Surg. 2005; 14(1 Suppl S): 99S-104S.

21. Sernik RA, Vidal Leão R, Luis Bizetto E, Sanford Damasceno R, Horvat N, Guido Cerri G. Thickening of the axillary recess capsule on ultrasound correlates with magnetic resonance imaging signs of adhesive capsulitis. Ultrasound. 2019; 27(3): 183-90.

22. Monkowsky D, Larese C. Bloqueo axilar del plexo braquial. Rev Arg Anest. 2004; 62(6): 440-4.

23. Oliveira C, Navarro García R, Navarro Navarro R, Ruiz Caballero JA, Jiménez Díaz JF, Brito Ojeda ME. Biomecánica del hombro y sus lesiones. Canarias Médica y Quirúrgica. 2007.

24. Lugo R, Kung P, Ma CB. Shoulder biomechanics. Eur J Radiol. 2008; 68(1): 16-24. doi: 10.1016/j.ejrad.2008.02.051.

25. López Espinosa OF, Pérez Solares A, Mejía Rohenes LC. Descripción del tipo de lesiones del manguito rotador más frecuentes en el Hospital Regional General Ignacio Zaragoza. Rev Esp Med Quir. 2008; 13(4): 173-6.

26. Halder AM, Itoi E, An KN. Anatomy and biomechanics of the shoulder. Orthop Clin North Am. 2000; 31(2): 159-76.

27. Zumstein MA, Jost B, Hempel J, Hodler J, Gerber C. The clinical and structural long-term results of open repair of massive tears of the rotator cuff. J Bone Joint Surg Am. 2008; 90(11): 2423-31. doi: 10.2106/JBJS.G.00677.

28. Desroches G, Aissaoui R, Bourbonnais D. Relationship between resultant force at the pushrim and the net shoulder joint moments during manual wheelchair propulsion in elderly persons. Arch Phys Med Rehabil. 2008; 89(6): 1155-61. doi: 10.1016/j.apmr.2007.10.040.

29. Ugalde Ovares CE, Zúñiga Monge D, Barrantes Monge R. Actualización del síndrome de hombro doloroso: lesiones del manguito rotador. Med Leg Costa Rica. 2013; 30(1): 63-71.

30. Kim IB, Jung DW. A rotator cuff tear concomitant with shoulder stiffness is associated with a lower retear rate after 1-stage arthroscopic surgery. Am J Sports Med. 2018; 46(8): 1909-18. doi: $10.1177 / 0363546518768813$.

31. Vega-Gutiérrez AE, Gómez-Pérez MG. Hill-Sachs lesion evaluated with magnetic resonance. Anales de Radiología México. 2014; 13(2): 136-42.

32. Kim IB, Jung DW, Suh KT. Prediction of the irreparability of rotator cuff tears. Arthroscopy. 2018; 34(7): 2076-84. doi: 10.1016/j. arthro.2018.02.033.

33. Leyes M, Forriol F. La rotura del manguito rotador: etiología, exploración y tratamiento. Trauma Fund MAPFRE. 2012; 23(1): 39-56.

34. Greenspoon JA, Millett PJ, Moulton SG, Petri M. Suppl 1: M2: Irreparable rotator cuff tears: restoring joint kinematics by tendon transfers. Open Orthop J. 2016; 10: 266-76.

35. Cofield RH. What I've learned about open shoulder surgery. J Shoulder Elbow Surg. 2018; 27(10): 1920-4. doi: 10.1016/j.jse.2018.01.025.

36. Collin P, Matsumura N, Lädermann A, Denard PJ, Walch G. Relationship between massive chronic rotator cuff tear pattern and loss of active shoulder range of motion. J Shoulder Elbow Surg. 2014; 23(8): 1195-202. doi: 10.1016/j.jse.2013.11.019.

37. Werner C, Steinmann P, Gilbart M, Gerber C. Treatment of painful pseudoparesis due to irreparable rotator cuff dysfunction with the Delta III reverse-ball-and-socket total shoulder prosthesis. $J$ Bone Joint Surg Am. 2005; 87(7): 1476-86.
38. Rössler H. Ruptures in the rotator aponeurosis (author's transl). Z Orthop Ihre Grenzgeb. 1976; 114(3): 282-94.

39. Ferreiro I, Veiga M, Guerra JL, Rey S, Paz J, Tobío A. Tratamiento rehabilitador del hombro doloroso. Rehabilitación (Madr). 2005; 39(3): 113-20.

40. Casal JR, Vázquez MJ. Abordaje del dolor musculoesquelético en urgencias. Emergencias. 2012; 24: 59-65.

41. Zas V, Rodríguez JR, Jiménez ES. El dolor y su manejo en los cuidados paliativos. Panorama Cuba y Salud. 2013; 8(2): 41-8.

42. Murphy RJ, Carr AJ. Shoulder pain. BMJ Clin Evid. 2010; 2010: 1107.

43. Artus M, van der Windt DA, Afolabi EK, Buchbinder R, Chesterton LS, Hall A, et al. Management of shoulder pain by UK general practitioners (GPs): a national survey. BMJ Open. 2017; 7(6): e015711. doi: 10.1136/bmjopen-2016-015711.

44. Kulkarni R, Gibson J, Brownson P, Thomas M, Rangan A, Carr AJ et al. Subacromial shoulder pain. Shoulder Elbow. 2015; 7(2): 135-43. doi: $10.1177 / 1758573215576456$.

45. Ranalletta M, Rossi LA, Bongiovanni SL, Tanoira I, Elizondo CM, Maignon GD. Corticosteroid injections accelerate pain relief and recovery of function compared with oral NSAIDs in patients with adhesive capsulitis: a randomized controlled trial. Am J Sports Med. 2016; 44(2): 474-81. doi: 10.1177/0363546515616238.

46. Srour F, Barette G, Loubiere M. Rehabilitación del hombro doloroso no operado ni inestable. EMC-Kinesiterapia-Medicina Física. 2015; 36(4): 1-21.

47. Suárez-Sanabria N, Osorio-Patiño AM. Biomecánica del hombro y bases fisiológicas de los ejercicios de Codman. CES Med. 2013; 27(2): 205-17.

48. Pogorzelski J, Fritz EM, Godin JA, Imhoff AB, Millett PJ. Nonoperative treatment of five common shoulder injuries. Obere Extrem. 2018; 13(2): 89-97. doi: 10.1007/sł1678-018-0449-1.

49. Levy O. Deltoid re-education program for massive rotator cuff tears. OpTechn Orthop. 2018; 28(4): 179-90.

50. Lu XW, Verborgt O, Gazielly DF. Long-term outcomes after deltoid muscular flap transfer for irreparable rotator cuff tears. J Shoulder Elbow Surg. 2008; 17(5): 732-7. doi: 10.1016/j.jse.2008.02.022.

51. Rubio-Oyarzún D, Araya-Quintanilla F, Gutiérrez-Espinoza H, Olguín-Huerta C, Fritz Y, Arias-Poblete L. Terapia de juicio de lateralidad e imaginería de movimiento y ejercicios de activación muscular selectiva glenohumerales en sujetos con ruptura masiva del manguito rotador: serie de casos. Rev Soc Esp Dolor. 2018; 25(4): 197-206.

52. De Mey K, Danneels L, Cagnie B, Huyghe L, Seyns E, Cools AM. Conscious correction of scapular orientation in overhead athletes performing selected shoulder rehabilitation exercises: the effect on trapezius muscle activation measured by surface electromyography. J Orthop Sports Phys Ther. 2013; 43(1): 3-10. doi: 10.2519/ jospt.2013.4283.

53. Riboh JC, Garrigues GE. Early passive motion versus immobilization after arthroscopic rotator cuff repair. Arthroscopy. 2014; 30(8): 9971005. doi: 10.1016/j.arthro.2014.03.012.

54. Sabari JS, Maltzev I, Lubarsky D, Liszkay E, Homel P. Goniometric assessment of shoulder range of motion: Comparison of testing in supine and sitting positions. Arch Phys Med Rehabil. 1998; 79(6): 647-51.

55. Naveed MA, Kitson J, Bunker TD. The Delta III reverse shoulder replacement for cuff tear arthropathy: a single-centre study of 50 consecutive procedures. J Bone Joint Surg Br. 2011; 93(1): 57-61. doi: 10.1302/0301-620X.93B1.24218.

56. Hantes ME, Venouziou AI, Liantsis AK, Dailiana ZH, Malizos KN. Arthroscopic repair for chronic anterior shoulder instability: a comparative study between patients with Bankart lesions and patients with combined bankart and superior labral anterior posterior lesions. Am J Sports Med. 2009; 37(6): 1093-8. doi: 10.1177/0363546508331139.

57. Hartzler RU, Barlow JD, An KN, Elhassan BT. Biomechanical effectiveness of different types of tendon transfers to the shoulder for external rotation. J Shoulder Elbow Surg. 2012; 21(10): 1370-6. doi: 10.1016/j.jse.2012.01.026. 